\title{
Technologies for Traceability in Inventory Management System
}

\author{
Mayuri Jamkhedkar ${ }^{1}$, Parth Sanghavi ${ }^{2}$, Pooja Gajera ${ }^{3}$, Prof. V. A. Mishra ${ }^{4}$ \\ Prof. K. H. Wanjale \\ Department of Computer Engineering, Vishwakarma Institute of Information \\ Technology, Pune, India \\ ${ }^{1}$ mayuri.17u301@viit.ac.in \\ 2parth.21820135@viit.ac.in \\ 3pooja.21820002@viit.ac.in \\ Co-Author: ${ }^{4}$ vaishali.mishra@viit.ac.in \\ Co-Author: ${ }^{5}$ kirti.wanjale@viit.ac.in
}

\begin{abstract}
Our main aim in this paper is to show that traceability has become more than way to guarantee the stock details but also useful after the delivery of the orders. In this paper we are trying to illustrate this idea where how companies can implement traceability using modern technologies. After researching with various industrial company about how traceability works, we came up the technologies which can help the company to implement traceability with ease. The companies needs traceability in order to track the products while packaging, before and after delivery of orders. With coherent traceability, it becomes easier locate the missing products in the orders after the delivery and provides credibility of the orders. We are going to review three technologies used in inventory management system and how can we use these technologies in traceability that is Barcode Technology, Radio Frequency Identification(RFID) and Near Field Communication(NFC).
\end{abstract}

Keywords: inventory management system, traceability, RFID, NFC, barcode technology

\section{Introduction}

At the moment we are in the very early stages, so it is difficult to put a specific time on the training, but we will keep people informed as plans are developed. The system is very user friendly and it is anticipated that functions of the system will be easily accessed by administrators and workers.

The term stock alludes to the merchandise or materials utilized by a firm with the end goal of creation and deal. It likewise incorporates the things, which are utilized as steady materials to work with creation. There are three essential kinds of stock: crude materials, work-in-progress and completed merchandise. Crude materials are the things bought by firms for use underway of completed item. Work-in-progress comprises of all things right now during the time spent creation. These are quite made items. Completed merchandise comprises of those things, which have effectively been created however not yet sold.

Stock traceability is frequently based on parts separated by a common trait: fabricate date, provider clump, fabric bunch serialized holders, and/or serialized person items. Genuine teach and (regularly manual) exertion is required to execute such part traceability. That exertion drives up the require for labour and increments labour costs. It is that fetched that regularly causes administration to address the esteem of stock traceability. Organizations inquire whether the forms around keeping up stock traceability are something for which their clients are willing to pay. Whereas clients don't continuously unequivocally state it, the overpowering prove is that stock traceability is an ever-increasing prerequisite in many businesses which it is getting to be a desire of providers. 
Within the past, stock traceability in manufacturing involved physically recording product details on paper holder labels and putting away those archives for recovery. Such strategies given moderate and wrong data in times of downstream deformity event. Advanced frameworks bolster these prerequisites through improved and coordinates Barcoding, filtering and program. RFID utilization continues to grow and the most excellent ERP arrangements back stock administration at the holder or person level. Through the benefits of such innovation, the fetched of stock traceability proceeds to diminish whereas time to follow significantly progresses. The innovation venture pays for itself by giving more exact data and moved forward stock turnover rates. The research focuses on the computerization of inventory management process through the use of web architecture and barcode technology. Barcode technology will use one-dimensional barcodes and remote corded barcode scanners. Barcode technology is superior to other technologies, such as radio frequency identification (RFID) and near field communication (NFC), because it is a mature, cheaper, and easierto-use technology.

\subsection{Motivation}

The motivation of this paper is to create better understanding in traceability and the importance it holds in today's industry. The value of traceability to prove credibility of the industry after the product deployment in case of some misunderstanding between the customer and company.

\subsection{Problem Statement}

In companies warehouse, the delivery of the orders is one of the most important process. Even after the delivery it is important to have traceability in the orders packed to give the credibility if all the items or the products are packed or not according to the given order. If it is a manual inventory system, the items will be lost due to human error in the procurement process, ultimately causing the entire order to suffer. Due to the lack of proper automation, its can cause the company's credibility in terms delivery of the order.

\subsection{Objectives}

1. Improvement: The main goal of the inventory management system is to improve and update the existing system to increase its efficiency and effectiveness. The software improves working methods by replacing the existing manual system with a computerbased system.

2. Automation: The inventory management system automates every activity of the manual system and improves its performance. Therefore, the response time of the system is very short and the running speed is very fast.

3. Accuracy: The inventory management system provides users with a quick response to very accurate information about users, etc. Any detail or system can be displayed exactly as needed.

4. User Friendly: Inventory management system software has a very user-friendly interface. Therefore, the user will feel very easy to operate it. The software provides precision and a nice interface. Make the current manual system more interactive, faster and easier to use. 
5. Availability: Can retry the system transaction report when required. Therefore, there is no delay in the availability of any information, and any information can be captured very quickly and easily. maintenance

\section{Costs: Reduces maintenance costs.}

\section{Barcode Technology}

Barcode technology is used for the computerization of operations in various fields of applications. The purpose of is to use a scanner to scan the import and export of commodities with a barcode, so as to achieve efficiency, effectiveness and optimal business income. The technology consists of a barcode and a barcode reader, also known as the scanner.

A barcode is a printed machine-readable data symbol that contains information about the item to help facilitate identification and tracking of the item. Modern inventory management software tends to be more streamlined, less complex, has a web interface and will allow you to use your smartphone/2D barcode to manage inventory. Advances in technology have had a huge impact on barcodes, and the market offers a number of different options. Perhaps the biggest breakthrough in barcode technology was the invention of 2D barcodes, which is a huge improvement over the 1D barcodes used previously. Twodimensional or 2D barcodes are used to systematically describe data using two-dimensional symbols and shapes. They have the ability to store more information such as prices, product quantities, product website web addresses, and even images, than their 1D counterpart. 2D barcodes have the advantage of containing more data per unit area and therefore can help make better business decisions. A barcode reader is an electronic instrument that scans barcodes printed on items for sale or other labels for identification. It is used to extract optical information from barcodes. By storing the data in the barcode for the orders packed in another, we can implement traceability for tracing the orders packed or not.

The advantages of barcode technology include: because the two main components used to produce barcodes are paper and ink, barcodes are therefore relatively cheaper than with RFID technology using chips made of silicon, it is easy to use, it is scalable, it is more reliable and accurate than manual data, and it provides real-time information.

The drawbacks of barcode technology include: Since barcode readers use a direct line of sight to the printed barcode when scanning the barcode, this makes the technology difficult and unheard of. practical in other applications. Industrial environments vary and conventional barcodes can only store a small amount of static information, usually around 20 characters.

\section{Radio Frequency Identification}

RFID is an automatic system that utilizes wireless technology to uniquely identify and track tagged objects within the range of a unique serial number. It gathers data about an object without the necessity to the touch or see the data carrier. A typical RFID system consists of 4 basic components including RFID tags, readers, antennas and a central node automatic processing system which houses the database server and management software. The RFID tag or transponder is that the information carrier that transmits information to the RFID reader (transceiver) within a given range through a microchip and antenna embedded in it. The second component in an RFID system is that the reader. Its role is to question a 
tag and receive data from it. The antenna generates radio signals to activate the tag and skim or write data thereto. The middleware at the central node manages incorporation of data received from the reader. The middleware manages the knowledge exchange between the reader and thus the backend database server. A electronic information service server mainly completes the knowledge storage, management and read-write control of the frequency tags. they provide the knowledge obtained from the reader to the software application.

Using RFID tags for inventory management offers several benefits, like reduced labour costs and faster scanning. Here's a glance at how RFID tags is a benefit within the inventory management process. They will even be read in any orientation and provides you improved visibility into your inventory with the potential for more frequent updates and scanning locations. With labour costs accounting for the maximum amount as $50-80 \%$ of distribution centre costs, RFID offers potential benefits during this area. Inventory check-in, counting, and shipment verification will be done very quickly and automatically in an exceedingly few scans without the necessity for multiple employees to process them.

The drawbacks of using the RFID are although there are fixed and remote RFID readers available, it's uphill to use a phone to scan them, as will be finished barcodes. RFID tags cost significantly over barcode labels. additionally, they utilize specific readers that has to be purchased from the limited number of RFID equipment manufacturers. this could add significant costs when scaling these solutions with the necessity for added specialized scanners and RFID tags. Setup for these systems requires the combination of the readers, tags, inventory management system, network, and building wiring which will take a major amount of your time and resources to line up. Also, if real-time asset tracking is required, the RFID-enabled system will have to utilize GPS and cellular data to speak, which may put a big burden on your system. While RFID systems still update and improve their data security, they will still be at risk of hacking. Remote devices, including cell phones, can sometimes be accustomed scan tags at close range and replica tag data. this might later be accustomed create a cloned tag or copy the data to a different tag, a risk of particular concern within the retail industry.

\section{Near Field Communication}

Near-field communication (NFC) could be a short-range wireless technology that produces your smartphone, tablet, wearables, payment cards, and other devices even smarter. Nearfield communication is that the ultimate in connectivity. With NFC, you'll be able to transfer information between devices quickly and simply with one touch-whether paying bills, exchanging business cards, downloading coupons, or sharing an enquiry paper. NFC tags are passive devices, which implies that they operate without an influence supply of their own and are reliant on a vigorous device to return into range before they're activated. The trade-off here is that these devices can't really do any processing of their own, instead they're simply accustomed transfer information to a vigorous device, like a smartphone. In order to power these NFC tags, electromagnetic induction is employed to make a current within the passive device. We won't get too technical on this, but the essential principle is that coils of wire may be accustomed produce electromagnetic waves, which may then be picked up and turned back to current by another coil of wire. this is often very kind of like the techniques used for wireless charging technologies, albeit much less powerful.

NFC makes it easy for users to create instant payments with their smartphone or tablet. you'll use a mobile wallet, like those offered by Google or Apple, to process a transaction 
with just some taps - no have to even open your wallet. It's also a highly versatile platform, therein it covers a variety of services and industries. Customers are better served through convenient, hassle-free technologies, which successively improves brand reputation and increases productivity.

\section{System Design}

The system objectives outlined in the feasibility study are the basis for starting the system design work. Many of the activities involved in this stage are technical and require a certain degree of experience in systems design, a complete understanding of computer-related technologies, and an understanding of commercially available computers and the various facilities provided by the system. seller. However, without the active participation of users, the system cannot be designed in isolation. At this stage, users also play a fundamental role. We know that the data collected during the feasibility study will be used systematically in the system design process. However, it should be remembered that a detailed study of the existing system does not necessarily end with the completion of the feasibility study. According to the feasibility study plan, the level of detailed study will be different, and the number of investigations in the system design phase will also be different, which still needs to be done. This investigation is usually an emergency activity during the system. Sometimes (but rarely), the investigation can form a separate stage between the feasibility study and the design of the computer system. Designing a new system is a creative process that requires logic and lateral thinking. A reasonable approach involves taking into account the capabilities of staff and teams at each step of decision-making and moving towards the systematization of the final product. Lateral thinking means including ideas beyond conventional functions and equipment. This is to ensure that no effort is made to adapt the old solution to the new situation.

\section{Solution}

\section{A. Barcode Technology:}

Using the Barcode Technology, the implementation of traceability is very basic. It's one of the most effective solutions in terms cost and usability. Supposedly, the company receives an order and once the order is packed, barcode will be sticked on it. The packages has various items where all the barcode are associated to each items. In this way, all the barcodes are linked to each other and the traceability is maintained. Once, the package reaches the customer, he can scan the package and get all the details regarding the contents and their production details which is connected to company ERP web architecture.

\section{B. Radio Frequency Identification}

Considering the same scenario, RFID takes care of the business's security and managements. RFID gets into picture whenever the items comes in the warehouse. Each item present within the consignment is tagged with an RFID IC chip, or sometimes, the full pallet is tagged with one chip. The chip contains information about the item, where it's been shipped from, what it contains, manufacturing details, etc. because the objects experience the warehouse, the info inside the RFID tag is updated. You can overwrite and browse the knowledge present inside the tag through an RFID reader. These devices are available different forms; handheld and stuck. you'll be able to choose whichever device suits you best. the knowledge present within the RFID tag is registered during a database located within the warehouse's network. The database is updated through RFID technology as soon as a replacement shipment arrives at the warehouse. 


\section{Near Field Communication}

This system model for NFC based on the internal control system for an electronic store. It uses a system of Internet of things and uses NFC tags or barcodes for interaction with the system along with the NFC end-device. Each product within the store contains an NFC passive tag. An NFC passive tag is sort of a paper sticker, which is intended in such the simplest way that it contains an NFC chip and therefore the antenna. NFC passive tags are called passive tags because they are doing not have their own power to operate; instead they're powered by the magnetic flux generated by the active tag (NFC reader). An energetic tag is termed active because it's its own power to operate; that may be battery or USB powered depending upon the kind of the reader.

For example, the company can attach a NFC tag on the package and transport it to the customer. The customer can tap the tag and the end-device which can be a mobile can give all the details regarding the content in the package resulting in the traceability of the packed packages within the order.

\section{Conclusion}

The request for inventory precision is more vital presently than ever some time recently. Consider the chance of losing clients who are requesting upgraded traceability. This paper creates an alarm for introducing the traceability after the dispatchment of the orders. As a matter of reality, for this company, traceability constitutes a blood circulation framework. As of nowadays, traceability is $100 \%$ operational and works perfectly well and certainly has ended up a source of competitive preferences. This project will minimize the risk between the customer and the company in terms of mishap in the orders. A framework with great stock traceability capabilities can arm you with the devices you wish to meet the desires of your clients and providers. The three technologies have their own pros and cons. Utilizing a RFID, it can improve exactness and can be proficient in carrying out traceability. The frameworks dependent on scanner tags in light of the restrictions can be less effective when contrasted with the frameworks dependent on NFC. Various analyses have shown that NFC has more noteworthy precision and lucidness when contrasted with scanner tags since standardized identifications should be perused at a legitimate point. Moreover, expansion in the capacity limit of NFC doesn't influence the size of the tag, which is one of the shortcomings of the standardized tag. This increment in the size of the standardized identification fundamentally lessens the clarity of scanner tags labels. Nonetheless, on account of NFC, regardless of whether the capacity limit of the tag is expanded, the size of the label stays unaltered. Security is another factor, which settles on NFC a superior decision in stock control frameworks than the standardized identification based stock control frameworks, since NFC has numerous layers of safety, which keeps the information secure and hidden if necessary to be. These all credits of NFC settle on it's a decent decision to be utilized in stock control frameworks. Both the RFID and NFC settles on a superior decision as far as security and the productivity of the board.

\section{Acknowledgement}

Special thanks to "Fluid Controls", a leading instrumentation provider company in Pune and Mr.Kiran Matkar for guiding us with new technologies and workflows for the project and the research. 


\section{References}

[1] Demian, Horia. (2009). Traceability In Stock Management Systems. Annals of Faculty of Economics. 4. 927-930

[2] S.Angel Raphella, S.Gomathi Nathan and G.Chitra, "Inventory Management- A Case Study", International Journal of Emerging Research in Management \&Technology, ISSN: 2278-9359, Vol.3 (3) June 2014, pp.94102.

[3] Alfaro, José \& Rbade, Luis. (2009). Traceability as a strategic tool to improve inventory management: A case study in the food industry. International Journal of Production Economics. 118. 104-110. 10.1016/j.ijpe.2008.08.030.

[4] Souvik Paul, Atrayee Chatterjee, and Digbijay Guha, "Study Of Smart Inventory Management System Based On The Internet Of Things (IOT)", International Journal on Recent Trends in Business

[5] Mohd Saad, Norhashimah. (2013). Barcode Recognition System. International Journal of Emerging Trends \& Technology in Computer Science (IJETTCS) 2278-6856. 2. 1-6.

[6] Williams, Brent \& Tokar, Travis. (2008). A Review of Inventory Management Research in Major Logistics Journals. The International Journal of Logistics Management. 19. 212-232. 10.1108/09574090810895960.

[7] Tersine, Richard. (1994). Principles of Inventory and Materials Management.

[8] Khan, F. and Siddiqui, D. A. (2019). Impact of Inventory Management on Firm's Efficiency - A Quantitative Research Study on Departmental Stores Operating in Karachi. Social Science and Humanities Journal, 3(4), 964-980

[9] AkramRoshdi, and AkramRoohparvar, "Information Retrieval Techniques and Applications , "International Journal of Computer Networks and Communications Securityvol. 3, no. 9, september 2015.

[10] Campos, Julio \& Miguez, Luis. (2009). Manufacturing traceability data management in the supply chain. IJITM. 8. 321-339. 10.1504/IJITM.2009.024608.

[11] Subrahmanian, Eswaran \& Rachuri, Sudarsan \& Fenves, Steven \& Foufou, Sebti \& Sriram, Ram. (2005). Product lifecycle management support: A challenge in supporting product design and manufacturing in a networked economy. International Journal of Product Lifecycle Management. 1. 10.1504/IJPLM.2005.007342.

[12] A. Acilar and Karamasa, "Factors Affecting the E-Commerce Adoption by Small Hotels: A Comparative Case Study,” International Journal of E-Adoption (IJEA), vol. 4, no. 1, pp. 1-10, 2012.

[13] K. A. Sarika and S. R. Imran, ” Effective Survey On Two-Dimension Color Barcodes For Mobile Applications," International Journal of Scientific Engineering and Applied Science (IJSEAS), vol. 2, no. 1, pp. 195-198, January 2016.

[14] Patel, Dr.Jignesh \& Kothari, Badal. (2013). Near Field Communication - The Future Technology For An Interactive World. International journal of Engineering Research and Science \& Technology. 2. 55-59.

[15] Saeed, Dawer \& Iqbal, Razi. (2015). NFC and Wi-Fi enabled smart clock for smart communication. 3035. 10.1109/ICOSST.2015.7396398.

[16] Iqbal, Razi \& Saeed, Muhammad \& Ahmad, Awais. (2014). Wearable Near Field Communication Ring Antenna for Mobile Communication.

[17] Taguinod, Cedie Elroy \& Chua, Pocholo \& Aragones, Calvin Jex \& Alcazar, Ed Carlo \& Godoy Jr, Carlo. (2021). Development of Inventory Management System. 